\title{
Function of BMP4 in the Formation of Vasculogenic Mimicry in Hepatocellular Carcinoma
}

\author{
Xiao Li1,2, Baocun Sun ${ }^{1,2}{ }^{\circledR}$, Xiulan Zhao ${ }^{1,2}$, Jindan An ${ }^{2,3}$, Yanhui Zhang ${ }^{3}$, Qiang Gu ${ }^{1,2}$, Nan Zhao ${ }^{1,2}$, Yong \\ Wang ${ }^{3}$, Fang Liu ${ }^{1,2}$ \\ 1. Department Of Pathology, General Hospital Of Tianjin Medical University, Tianjin, 300052, China \\ 2. Department Of Pathology, Tianjin Medical University, Tianjin, 300070, China \\ 3. Department of Pathology, Cancer Hospital of Tianjin Medical University, Tianjin, 300060, China \\ $\triangle$ Corresponding author: Baocun Sun, General Hospital and Department of Pathology Of Tianjin Medical University, Tianjin, 300052, China. \\ E-mail:baocunsun@aliyun.com.
}

(c) The author(s). This is an open access article distributed under the terms of the Creative Commons Attribution License (https://creativecommons.org/licenses/by/4.0/). See http://ivyspring.com/terms for full terms and conditions.

Received: 2019.09.23; Accepted: 2020.01.21; Published: 2020.02.10

\begin{abstract}
Vasculogenic mimicry (VM) is linked to vascular invasion of human hepatocellular carcinoma (HCC). BMP4, one BMP family member, is upregulated in several cancers. The purpose of this report is to identify the function of BMP4 in the formation of VM in HCC and the mechanism underling this regulation. In our report, BMP4 up-regulation resulted in an increase in migration, invasion and channel-like structure formation as well as induced epithelial-mesenchymal transition (EMT) process and stem cell-associated proteins OCT4 and SOX2 expression in HCC cells. In addition, The VM-associated proteins, including EphA2, VE-cadherin and MMP2, also could be effectively enhanced by the overexpression of BMP4. Furthermore, according to the TCGA database, higher expression of BMP4 is seen in HCC in contrast to normal liver samples. Immunohistochemistry revealed that BMP4 was positively associated with VM formation, age, histological differentiation, HCC stage, and shorter survival duration. These data demonstrated that BMP4 could promote VM network formation in HCC through induction of stemness in EMT and modulating the EphA2/VE-cadherin/MMP2 signaling pathway.
\end{abstract}

Key words: Bone morphogenetic protein 4, hepatocellular carcinoma, vasculogenic mimicry, epithelialmesenchymal transition, stemness

\section{Introduction}

Hepatocellular carcinoma (HCC) has a high incidence worldwide [1, 2]. It is frequently linked to vascular invasion and high metastasis [3]. Previous studies of our team have demonstrated that vasculogenic mimicry (VM) has a significant impact on HCC blood supply and is essential for HCC cell survival [4]. VM, unlike traditional angiogenesis, describes the formation of blood vessels by highly invasive tumor cells [5]. Patients who display VM have more invasive cancer phenotypes [6]. A number of VM-associated proteins have been identified, including EphA2, VE-cadherin, MMP2, OCT4 and SOX2 [7-9]. Studies have also demonstrated that VM in tumor is linked to epithelial-mesenchymal transition (EMT) and stem cell-like properties [10, 11].
A signaling pathway of the VM was first proposed by Hendrix and colleagues [12], which suggested that VE-cadherin and EphA2 colocalize and subsequently lead to activation of matrix metalloproteinase 2 (MMP2) [13]. Nevertheless, the exact underlining mechanism in VM formation in HCC requires further analysis.

Bone morphogenetic proteins (BMPs) are part of the transforming growth factor $\beta$ (TGF- $\beta$ ) superfamily. Upon binding to the associated membrane receptor, BMPs phosphorylate and activate Smad1/5/8 protein complex, which assemble with SMAD4 and activate or suppress downstream targets $[14,15]$. BMPs have significant effects on cell proliferation, differentiation and embryonic 
development [14]. In addition, researchers have claimed that BMPs also play a variety of roles in carcinomas [16-21]. One BMP family member, BMP4, is essential for the morphogenesis of hepatic diverticulum, also known as a liver bud [22-24]. Studies have indicated that BMP4 enhances invasion and metastasis [25-29]. In particular, BMP4 serves as an angiogenic factor that promotes vascular network formation in HCC $[26,30]$. More interestingly, BMP4 is critical in epithelial-mesenchymal transition (EMT) process, which possesses stem-cell like characteristics [31-35]. Moreover, EMT and stem-like features are both involved in VM [10, 11]. Research found that BMP4 is linked to VM channel formation, which appears to be mediated through increasing VE-cadherin and EphA2 expressions [25]. Another study also supported that BMP4 increases MMP2 expression [36], which is involved in $\mathrm{VM}$ and the downstream EphA2/ VE-cadherin/ MMP2 pathway [12]. Based on the above data, it sparked our interest in exploring whether BMP4 could promote VM formation in HCC via inducing EMT stemness and modulating the EphA2/ VE-cadherin/ MMP2 pathway. In this report, we investigated the relationship between BMP4 and VM in HCC tumors and examined the effect of BMP4 on migration, invasion and VM formation in human HCC cell lines. Finally, we evaluated whether BMP4 could promote the formation of VM through induction of EMT stemness and modulating the EphA2/ VE-cadherin/ MMP2 pathway.

\section{Methods}

\section{Patient tissues}

Patient tissues were collected from 92 HCC patients receiving live resection in Tianjin Cancer Hospital and Tianjin General Hospital. We gathered exhaustive pathologic and clinical data of the 92 patients. These patients were never treated with antitumor therapy before the surgery. The protocol was approved by the Ethics Committee of Tianjin Cancer Hospital and Tianjin General Hospital.

\section{Immunohistochemical (IHC) staining}

Paraffin-embedded tumor samples $(4-\mu \mathrm{m})$ were deparaffinized, hydrated and treated with endogenous peroxidases. Then, the slides were subject to antigen retrieval. Next, the slides were subject to $10 \%$ normal goat serum to inactivate endogenous peroxidase and treated overnight with rabbit monoclonal against BMP4 antibody (ab124715, Abcam, USA) at $4^{\circ} \mathrm{C}$. Following incubation, the corresponding secondary antibody was added. Then, we used 3,3' -diaminobenzidine chromogen to detect peroxidase activity. Ultimately, slides were counterstained with hematoxylin. Staining result was assessed based on a previously described method [37].

\section{CD31/ Periodic Acid-Schiff (PAS) double staining and definition of VM}

IHC staining of CD31 (ZM-0014, Zhongshan Chemical Co., China) was applied as described above and slides were then subject to PAS, ultimately hematoxylin staining was performed.

$\mathrm{VM}$ is characterized based on previously established definition [38]. Briefly, VM is known to form channels under the tumor cell microenvironment in exhibiting red blood cells. VM is present in CD31/PAS double-stained sections but not CD31-stained sections.

\section{Cell lines}

HepG2 HCC cell line (American Type Culture Collection) and SMMC7721 HCC cell line (Zhongshan Hospital Affiliated to Fudan University, Shanghai, China) were passaged in DMEM (Neuronbc, China) supplemented with $10 \%$ fetal bovine serum (FBS) (Gibco, USA). Experiments were performed when cells were $70-80 \%$ confluent.

\section{Expression plasmids and cell transfection}

The expression plasmids containing BMP4 cDNA and BMP4 shRNA were obtained from GeneCopoeia, Inc. (catalog no. EX-A0242-M98; HSH053910-CU6). HepG2 cells were transfected with the BMP4 cDNA-containing plasmid to establish the HepG2-BMP4 up-regulation model; SMMC7721 cells were transfected with the BMP4 shRNA-containing plasmid to establish the SMMC7721-shBMP4 down-regulation model.

\section{Wound healing assay}

HepG2, HepG2-BMP4, SMMC7721, and SMMC7721-shBMP4 cells were cultured in six-well culture plates for $24 \mathrm{~h}$. Then, a plastic tip was used to scratch a straight line in each well. The speed of wound closure was assessed after $24 \mathrm{~h}$ and $48 \mathrm{~h}$.

\section{Invasion assay}

The invasion assay was applied in 24-well chambers with $8 \mu \mathrm{m}$ pores (BD Biosciences, USA). First, the top chambers were coated with Matrigel (BD Biosciences, USA). Then, $1 \times 10^{5}$ cells were subject to serum-free DMEM in the top chambers, while DMEM with FBS were subject to bottom chambers. After $48 \mathrm{~h}$ incubation, invading cells that added to the bottom were fixed and then subject to crystal violet staining. Finally, invading cells were counted and photographed (Nikon, Japanese). 


\section{Three-dimensional (3D) cultures}

96-well plates were coated with Matrigel $(35 \mu l$ per well) for $30 \mathrm{~min}$ at $37^{\circ} \mathrm{C}$. Then, HepG2, HepG2-BMP4, SMMC7721, and SMMC7721-shBMP4 cells in DMEM supplemented with 10\% FBS were seeded on the surface of solid gel for $24 \mathrm{~h}$ at $37^{\circ} \mathrm{C}$. Finally, the tube-like networks were assessed and photographed (Nikon, Japanese).

\section{Zymography assays}

Serum-free culture medium were clumped and subjected to sodium dodecyl sulfate polyacrylamide gel electrophoresis (SDS-PAGE). Gels were washed in $2.5 \%$ Triton X-100 then cultured with developing buffer overnight at $37^{\circ} \mathrm{C}$. Subsequently, the Coomassie Brilliant Blue R250 staining was performed. Finally, the gels were washed with water. The proteolytic activity of MMP2 was detected as clear bands on the gel.

\section{Western blot}

HepG2, HepG2-BMP4, SMMC7721, and SMMC7721-shBMP4 cells were lysed in 10\% SDS. Cell lysates were first subject to SDS-PAGE, transferred to polyvinylidene fluoride membranes (Millipore, USA) and then blocked in non-fat milk. Primary antibodies including BMP4 (ab124715, Abcam, USA), E-cadherin (zs-78700, Zhongshan Chemical Co., China), Vimentin (2707-1, Epitomics, USA), EphA2 (sc-924, Santa Cruz Biotechnology, USA), VE-cadherin (ab33168, Abcam, USA), MMP2 (sc-13595, Santa Cruz Biotechnology, USA), OCT4 (sc-8629, Santa Cruz Biotechnology, USA) and SOX2 (GTX101507, GeneTex, USA) were added to the membranes at $4^{\circ} \mathrm{C}$ overnight. The next day, the secondary antibodies including goat anti-rabbit IgG-HRP (ZB-2301, Santa Cruz Biotechnology, USA), goat anti-mouse IgG-HRP (ZB-2305, Zhongshan Chemical Co., China) and rabbit anti-goat IgG-HRP (ZB-2306, Zhongshan Chemical Co., China) were added for $2 \mathrm{~h}$ at $37^{\circ} \mathrm{C}$. The protein levels were assessed using the enhanced chemiluminescence method.

\section{Immunofluorescence staining}

Cells were seeded on coverslips, permeabilized with ice-cold methanol, and incubated with normal goat serum $(5 \%)$. The primary antibodies were maintained with the slips overnight at $4^{\circ} \mathrm{C}$. The next day, secondary antibodies were added for $2 \mathrm{~h}$ at $37^{\circ} \mathrm{C}$. After immunolabeling, 4',6-diamidino-2-phenylindole (DAPI; Zhongshan Chemical Co., China) was used for nuclei staining. Finally, the cells were mounted and photographed (Nikon, Japanese).

\section{Bioinformatics analysis}

TCGA (https://cancergenome.nih.gov/) database was performed to predict the expression levels of BMP4 in HCC and normal tissues. A protein-protein interaction (PPI) network of BMP4 correlated genes was constructed according to the STRING (https://string-db.org/) database.

\section{Statistical analysis}

All experiments applied at least in triplicate independently, results were expressed as mean \pm standard deviation (SD) and assessed using SPSS17.0 software. Two-sided Pearson Chi-square test and Student's t-test were performed to compare the outcomes. $\mathrm{P}<0.05$ was regarded as statistically significant.

\section{Results}

\section{BMP4 was overexpressed in human HCC samples and associated with VM and clinicopathological features}

To validate the effect of BMP4 on $\mathrm{HCC}$, we investigated BMP4 expression in HCC and normal liver samples by using the TCGA database. We observed that BMP4 was notably enhanced in HCC $(p<0.05$, Fig. 1A). Subsequently, we evaluated BMP4 expression in HCC using immunohistochemistry analysis. Studies present VM identification in HCC tissue using CD31-PAS double-staining [10, 39]. In 17 of $92(18.48 \%)$ HCC samples, VM vessels were observed to be lined by tumor cells without inflammatory cell infiltration or necrosis around the channels (Fig. 1B). Positive expression of BMP4 was displayed in the cytoplasm of HCC cells. According to the existence of BMP4, 92 samples were categorized into two teams: BMP4 positive staining team $(n=52)$ and BMP4 negative staining team $(\mathrm{n}=40)$ (Fig. 1C). Among the 52 BMP4-positive group, 14 (26.92\%, $14 / 52$ ) showed presence of $\mathrm{VM}$; and among 40 BMP4-negative group, $3(7.5 \%, 3 / 40)$ showed presence of VM. The outcome indicated that BMP4 was positively associated with VM ( $\mathrm{P}=0.017$, Table 1$)$. The colocalization of BMP4 expression and VM was identified by IHC in consecutive sections (Fig. 1D). Next, the correlation between BMP4 and pathological features were analyzed. Based on our findings, BMP4 was significantly correlated with age, histological differentiation, and cancer stage $(\mathrm{P}=0.004,0.044$, and 0.019 , respectively, Table 2 ). In patients over 45 years old, BMP4 was much higher than in samples from patients aged 45 years or younger $(65.67 \%$, 44/67 versus $32.00 \%, 8 / 25$, respectively). Among the four HCC histological differentiation and stage types, BMP4 was found at higher expression in III/IV than 
I/II types $(63.93 \%, 39 / 61$ versus $41.94 \%, 13 / 31$; $66.67 \%, 36 / 54$ versus $42.11 \%, 16 / 38$, respectively). However, the presence of BMP4 did not show any association with other pathological characteristics, such as gender, tumor size, or metastasis $(\mathrm{P}>0.05$, Table 1). To determine the prognostic role of BMP4, the Kaplan-Meier analysis was applied. Analysis showed that patients of positive BMP4 expression had poorer prognosis than patients of negative BMP4 expression $(\mathrm{P}=0.046$, Fig. 1E).

Table 1: The relationship between BMP4 and VM in HCC

\begin{tabular}{|c|c|c|c|c|c|}
\hline \multirow[t]{2}{*}{ Variant } & \multirow{2}{*}{$\frac{\text { Total }}{\mathrm{N}}$} & \multicolumn{2}{|l|}{ VM } & \multirow[t]{2}{*}{$x^{2}$} & \multirow[t]{2}{*}{ P value } \\
\hline & & $\begin{array}{l}\text { Negative } \\
(\%)\end{array}$ & Positive (\%) & & \\
\hline \multicolumn{6}{|l|}{ BMP4 } \\
\hline Negative & 40 & $37(92.5)$ & $3(7.5)$ & 5.662 & $0.017^{*}$ \\
\hline Positive & 52 & $38(73.08)$ & $14(26.92)$ & & \\
\hline
\end{tabular}

$P^{\star}<0.05$
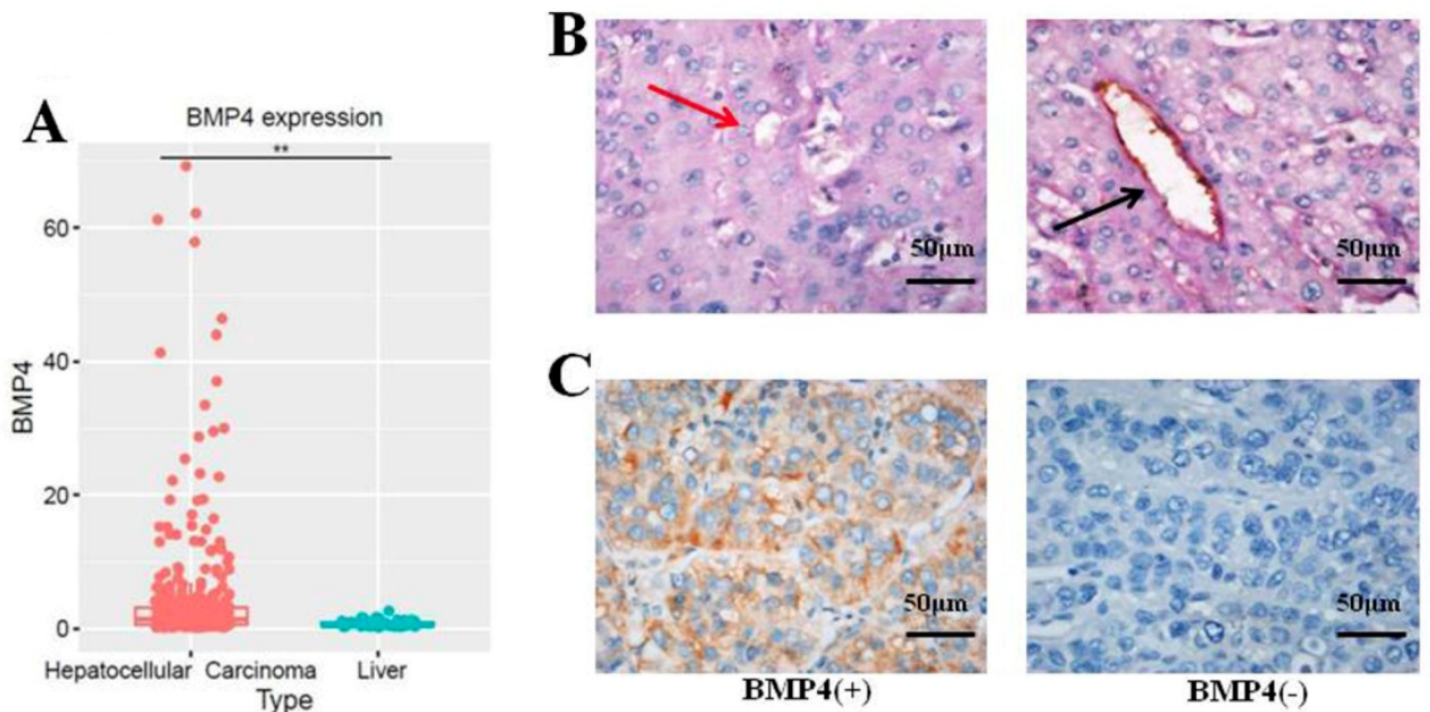

$\operatorname{BMP4}(+)$

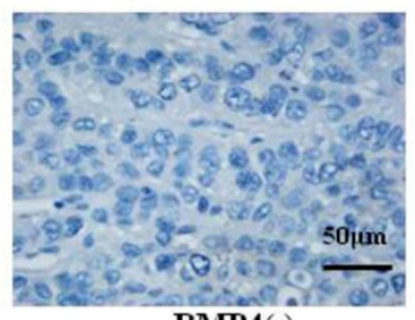

BMP4(-)

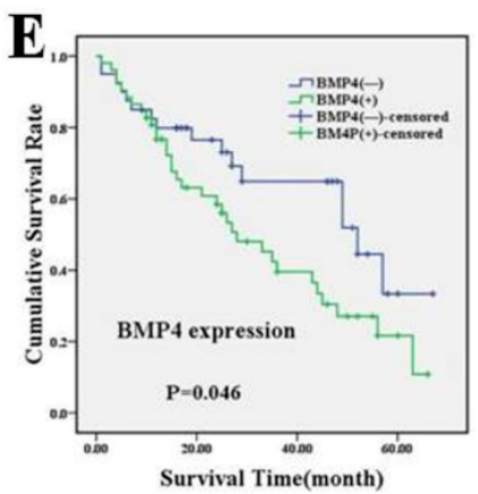

Fig. 1. (A) BMP4 was dramatically enhanced in $\mathrm{HCC}(n=374)$ in contrast to normal liver samples ( $n=50)$ according to the TCGA database. *P<0.05. (B) Evidence of $\mathrm{VM}$ (red arrow) and angiogenesis (black arrow) in HCC samples $(400 x$, scale bar $=50 \mu m)$. (C) BMP4 expression in HCC (400x, scale bar=50 $\mu$ m). BMP4 in VM-positive (left) and VM-negative (right) HCC samples. (D) Colocalization of BMP4 and VM formation was identified by IHC in consecutive sections (blue arrow, 400x, scale bar=50 $\mu \mathrm{m})$. (E) BMP4-positive patients had poorer survival compared to BMP4-negative patients according to Kaplan-Meier survival analysis $(\mathrm{P}=0.046)$. 
A

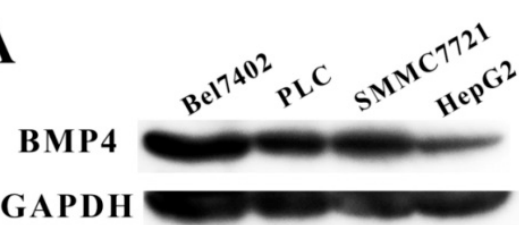

B
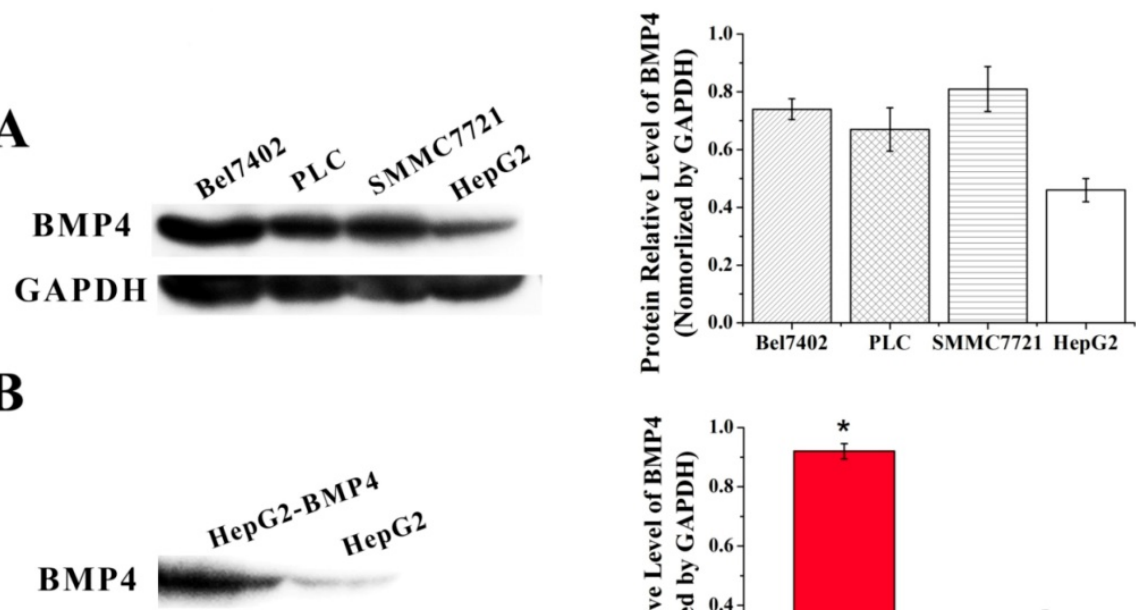

GAPDH
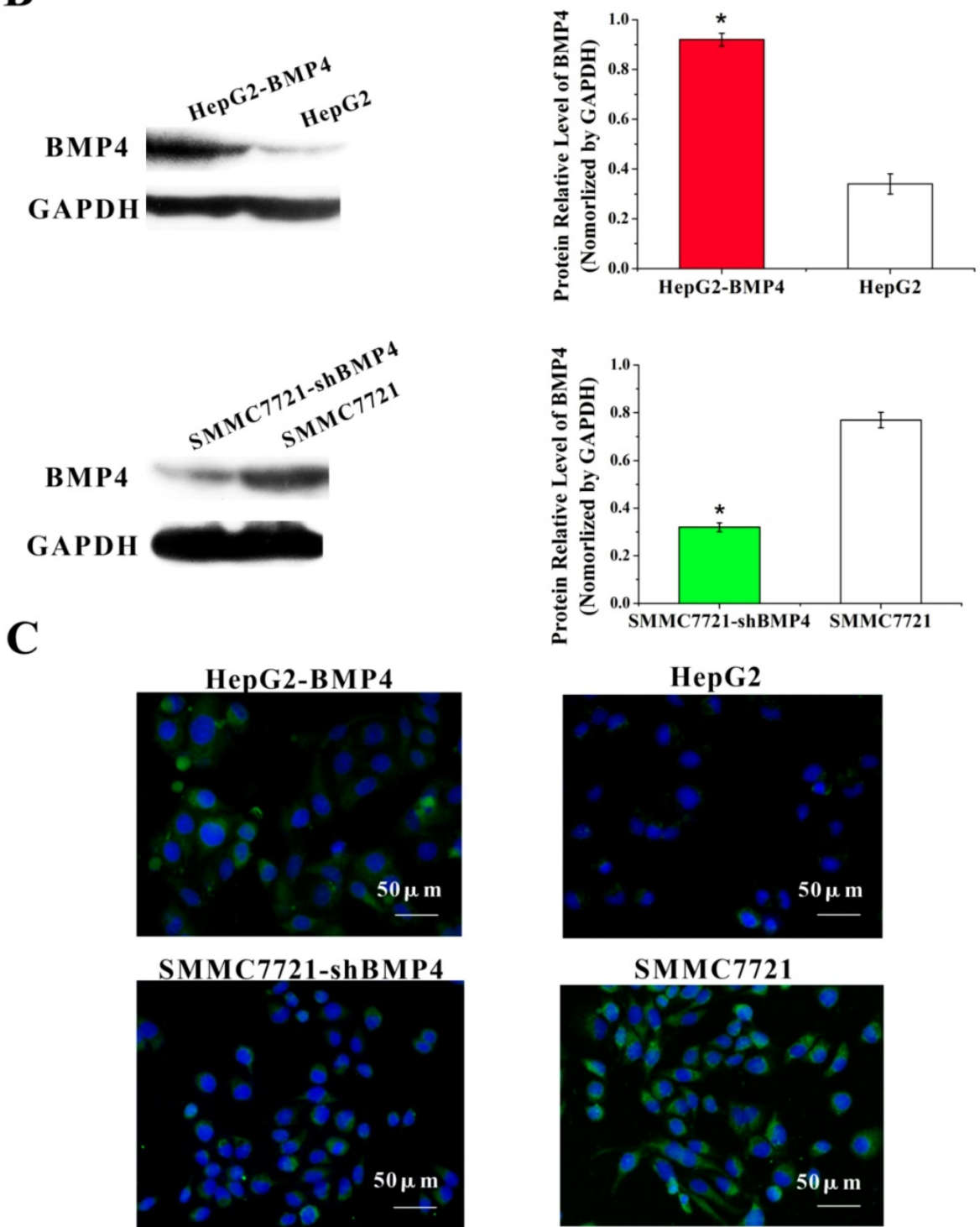

Fig. 2. (A) The expression of BMP4 in four HCC cell types was evaluated according to western blot analysis. (B, C) BMP4 protein level was up-regulated in BMP4-overexpressing HepG2 cells; the up-regulation efficiency was validated through western blot and immunofluorescence. BMP4 was knocked down in SMMC7721 cells using shRNA interference; the down-regulation efficiency was also validated through western blot and immunofluorescence. Green: target protein; blue: DAPI-stained nuclear DNA. *P<0.05, scale bar $=50 \mu \mathrm{m}$.

\section{BMP4 up-regulation in HepG2 cells and down-regulation in SMMC7721 cells}

First, we compared BMP4 protein expression in four HCC cell types. Based on western blot, we found that well-differentiated HepG2 cells had lower BMP4 expression compared to poorly differentiated SMMC7721 cells (Fig. 2A). After separated transfection of BMP4 cDNA in HepG2 cells and BMP4 shRNA in SMMC7721 cells, western blot (Figure. 2B) and immunofluorescence (Figure. 2C) confirmed that BMP4 expression in HepG2-BMP4 group was enhanced and BMP4 expression in SMMC7721shBMP4 group was decreased compared to their respective controls. 
Up-regulation of BMP4 enhanced HCC cells migration and invasion capabilities, while BMP4 down-regulation inhibited migration and invasion capabilities

The function of BMP4 in HCC cell migration and invasion were evaluated by the up-regulation and down-regulation cell culture systems described above. Wound healing assay was utilized for assessing cell migration. Compared with controls, BMP4 up-regulation resulted in faster wound healing while BMP4 down-regulation showed the opposite (Fig. 3A). These results support that BMP4 plays a positive role in cell migration. Similarly, following the invasion assay (Fig. 3B), invading cells were significantly enhanced in HepG2-BMP4 cells but reduced in SMMC7721-shBMP4 cells compared to respective controls. In conclusion, BMP4 up-regulation enhanced HCC cell migration and invasion capabilities, while BMP4 down-regulation inhibited HCC cell migration and invasion capabilities.

\section{Up-regulation of BMP4 induced VM formation and BMP4 down-regulation impaired VM formation}

A well-established 3D cell culture model was utilized for validating the effect of BMP4 expression on VM formation. In 3D Matrigel cultures, HepG2 cells failed to construct channel-like structures (Fig. 4B), but SMMC7721 cells were able to form such networks (Fig. 4D). We suspected that the lack of vascular network formation in HepG2 cells were due to low BMP4 expression. As expected, overexpression of BMP4 in HepG2 cells resulted in strong VM formation (Fig. 4A). Meanwhile, BMP4 down-regulation in SMMC7721 cells showed a dramatical suppression in pipe-like network formation (Fig. 4C). These data further indicated that BMP4 is an inducing factor of VM network formation in HCC cells.

\section{Up-regulation of BMP4 induced EMT and increased the expression of stem cell-associated proteins OCT4 and SOX2; down-regulation of BMP4 reversed EMT and decreased the expression of stem cell-associated proteins OCT4 and SOX2}

EMT is characterized by losing epithelial properties and taking on an invasive phenotype typical of mesenchymal cells [40]. EMT is previously thought to be associated with VM in HCC [10]. To validate the effect of BMP4 on EMT process, we examined changes in EMT-associated factors in HepG2-BMP4 and SMMC7721-shBMP4 cells (Fig. 5A, Fig. 5B). Results from HepG2-BMP4 cells indicated that there was a remarkable increase of vimentin and a great decrease of E-cadherin compared to HepG2 control cells. The opposing effect was observed in SMMC7721-shBMP4 cells. Taken together, we hypothesized that overexpression of BMP4 may induce EMT, while BMP4 knockdown may reverse EMT.

Cancer cells undergoing EMT display stem cell-properties including tumor- initiating and self-renewal [41, 42]. Thus, we examined stem cell regulators, OCT4 and SOX2, after BMP4 overexpression and knockdown. As expected, analysis revealed that up-regulation of BMP4 increased OCT4 and SOX2. Conversely, knockdown of BMP4 decreased OCT4 and SOX2 expression (Fig. 5A, Fig. 5B). Our results demonstrated that BMP4 may confer stem cell-like behaviors in HCC cells.

\section{BMP4 may lead to the formation of VM via the EphA2/VE-cadherin/MMP2 pathway}

Currently proposed signaling cascade for the formation of VM involves both EphA2 and VE-cadherin, which induce the expression and activation of MMP2 that ultimately result in VM formation [12]. EphA2 is a transmembrane receptor, which regulates cellular migration and invasion. Suppression of EphA2 expression impairs the formation of VM in vitro [43-45]. In our experiments, EphA2 was dramatically increased in HepG2-BMP4 cells, and the opposite result was found in SMMC7721-shBMP4 cells (Fig. 6A, Fig. 6B).

$\mathrm{VE}$-cadherin is critical in VM formation [46]. Remarkably, in HepG2-BMP4 group, VE-cadherin expression was effectively enhanced; while in SMMC7721-shBMP4 group, there was a down-regulation in VE-cadherin compared with control cells (Fig. 6A, Fig. 6B).

MMP2 is a key player in VM formation that acts as an effector molecule [12, 13, 47]. As shown in Fig. $6 \mathrm{~A}$ and Fig. 6B, MMP2 expression was strikingly enhanced after the up-regulation of BMP4 in HepG2 cells. The opposite result was found in SMMC7721-shBMP4 cells due to the down-regulation of BMP4. Furthermore, zymographic assay (Fig. 6C) results were in accordance with western blot and immunofluorescence. Based on the above data, HCC cells may enhance EphA2, VE-cadherin expression, and activate MMP2 via increasing BMP4 expression, which is ultimately promoting the formation of VM.

\section{Protein-protein interaction (PPI) network analysis}

To further validate the involvement of BMP4 in VM formation, we used the STRING database to construct a PPI network that identified interactions 
between BMP4 and 7 genes involved in VM formation. According to the network, BMP4 directly or indirectly interacted with VE-cadherin (CDH5), EphA2, MMP2, E-cadherin (CDH1), vimentin (VIM),

A
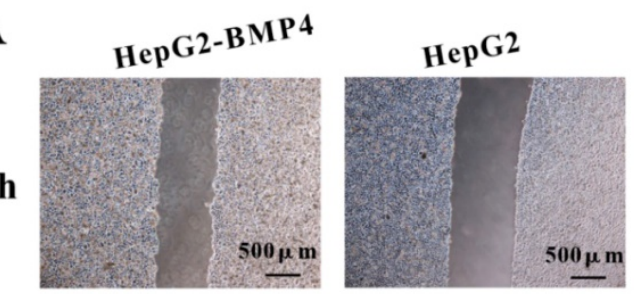

$24 h$
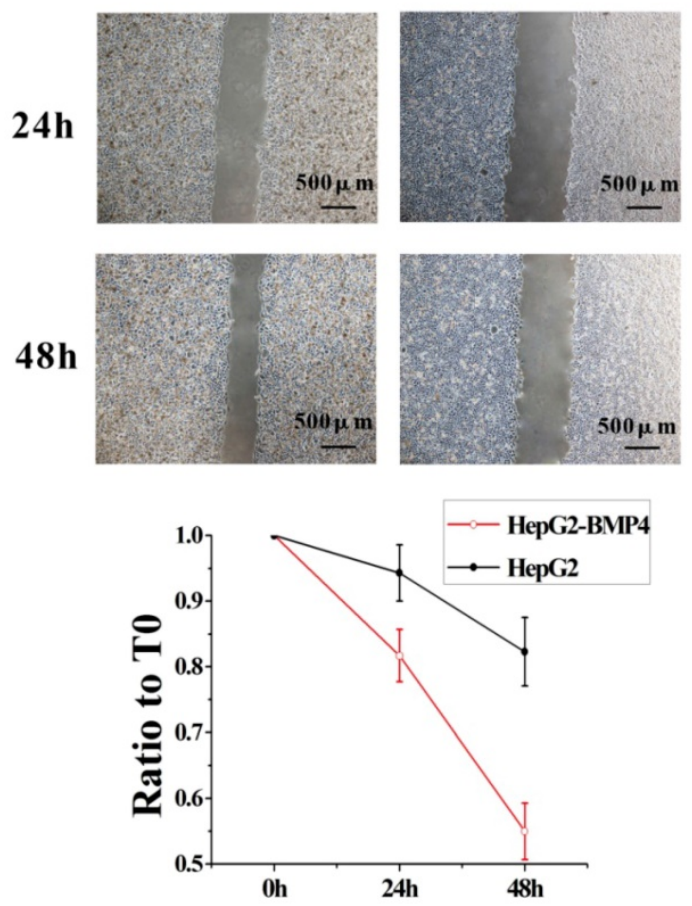

B
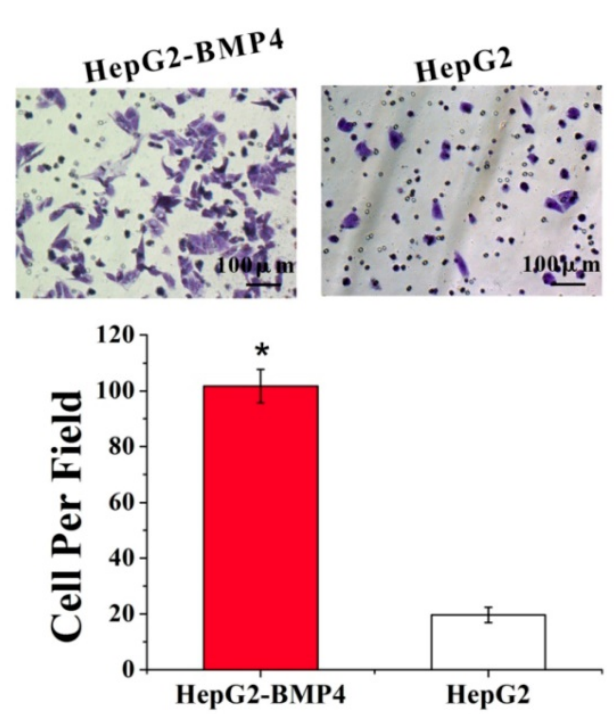

OCT4 (POU5F1) and SOX2 (Fig. 7). Thus, we speculate that BMP4 might promote VM formation through modulating these 7 genes.
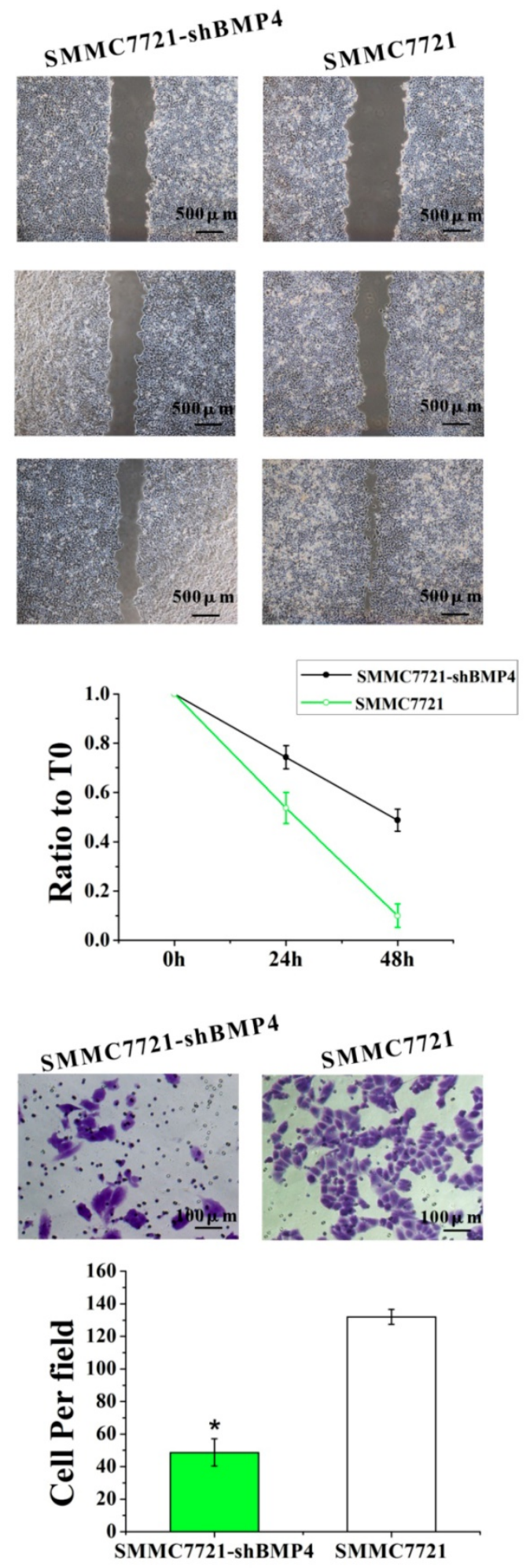

Fig. 3. The effects of BMP4 overexpression and knockdown in HCC cell migration and invasion. (A) The wound healing assay demonstrated that in contrast to controls, BMP4 up-regulation resulted in faster wound healing speed and BMP4 knockdown resulted in slower wound healing speed, scale bar=500 $4 m$. (B) In the invasion assay, BMP4 overexpression presented a greater increase in cell invasion compared with control HepG2 cells, and BMP4 knockdown presented a notable decrease in contrast to control SMMC7721 cells. $* \mathrm{P}<0.05$, scale bar $=100 \mu \mathrm{m}$. 
A

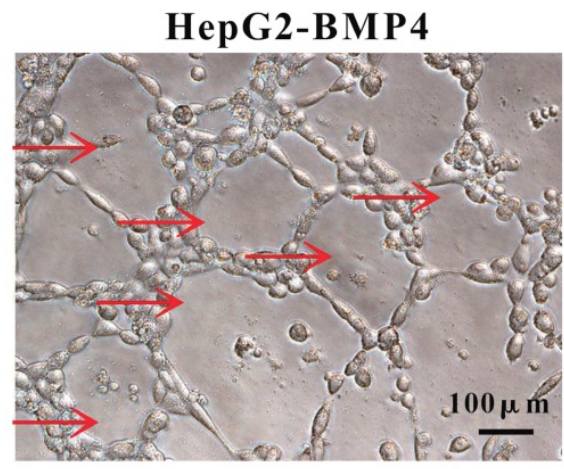

C

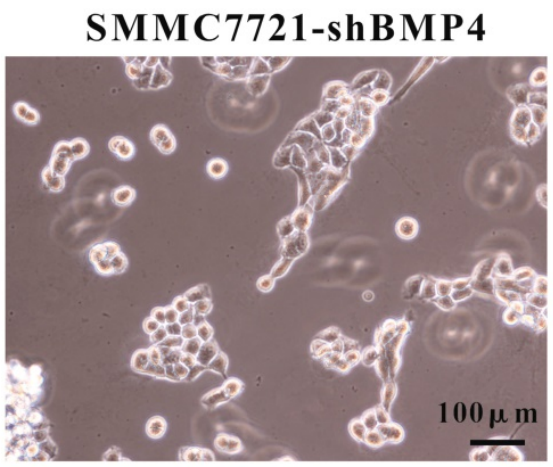

B

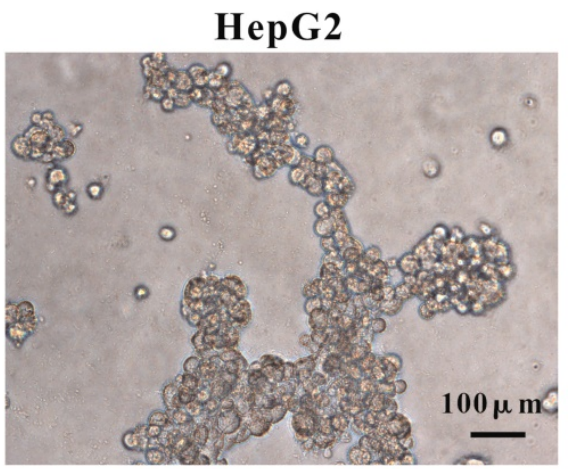

D

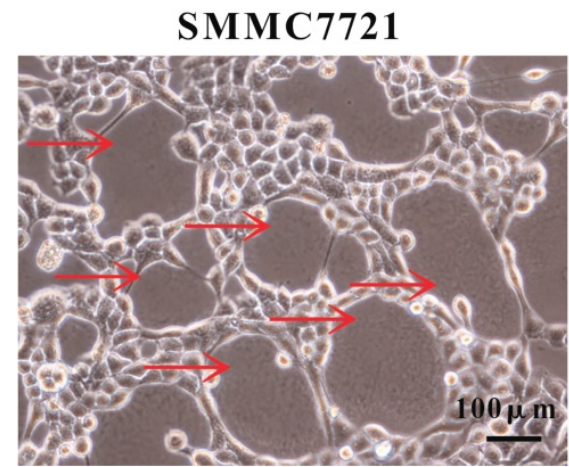

Fig. 4. VM formation in 3D cell culture. (A, B) The condition of VM structures in HepG2 up-regulation group and control group. (C, D) The condition of VM structures in

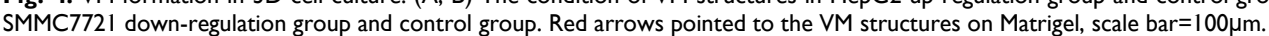

\section{Discussion}

This is the first study to report a connection between BMP4 and VM formation in HCC. The presence of VM signifies an alternative method for tumor cells to receive blood supply other than angiogenesis $[5,48]$. Previous reports have indicated that VM is related to the invasiveness and metastasis of various carcinomas $[6,49,50]$. In particular, VM formation is linked to short survival in HCC [4]. In our report, we observed that BMP4 was overexpressed in HCC samples and positively linked to age, histological differentiation, stage, and poor prognosis. Moreover, overexpression of BMP4 in HepG2 cells promoted migration, invasion and formation of 3D networks. We also found that down-regulation of BMP4 in SMMC7721 cells undermined the capabilities of migration, invasion and formation of VM channels. The accumulating evidence showed that BMP4 exerted an enormous influence on cell migration and invasiveness as well as the formation of VM in HCC. From HCC samples, we also observed that BMP4 expression was positively associated with VM networks. This result further demonstrated that the plasticity of some HCC cells, which are needed for VM formation, enhanced by BMP4 through a specific molecular pathway.

EMT, which loses epithelial abilities and obtains the mesenchymal properties, is known to be beneficial to invade, expand and disseminate in carcinomas. It is characterized molecularly by inhibition of E-cadherin and induction of vimentin [51-54]. Previous reports have suggested that the formation of VM is positively related to the EMT in HCC. In addition, a hypothesis was put forward to describe the similarities between tumor cell plasticity in a VM network to the embryonic vasculogenesis network formation [5]. Furthermore, some reports have demonstrated that the EMT possessed stem cell-like features. Thus, VM formation during EMT may also display stem-like characteristics. In the present study, overexpression of BMP4 in HepG2 cells led to EMT process as well as increased stem cell-associated protein OCT4 and SOX2. On the contrary, in SMMC7721-shBMP4 cells, EMT state was reversed and decreases in OCT4 and SOX2 expression were observed. Therefore, we concluded that BMP4 could induce VM formation by promoting EMT process and increasing stem cell-related protein expression. 


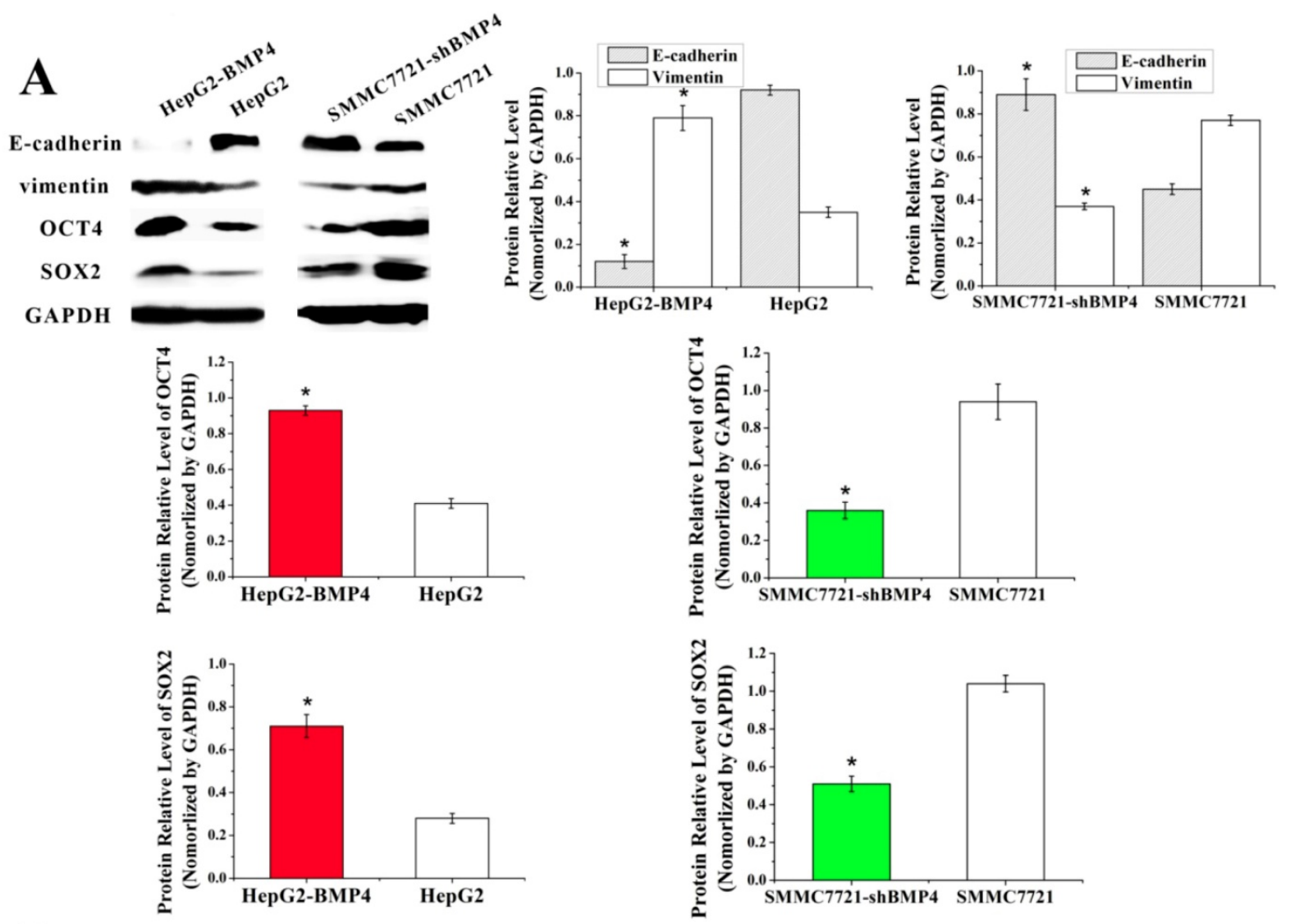

B
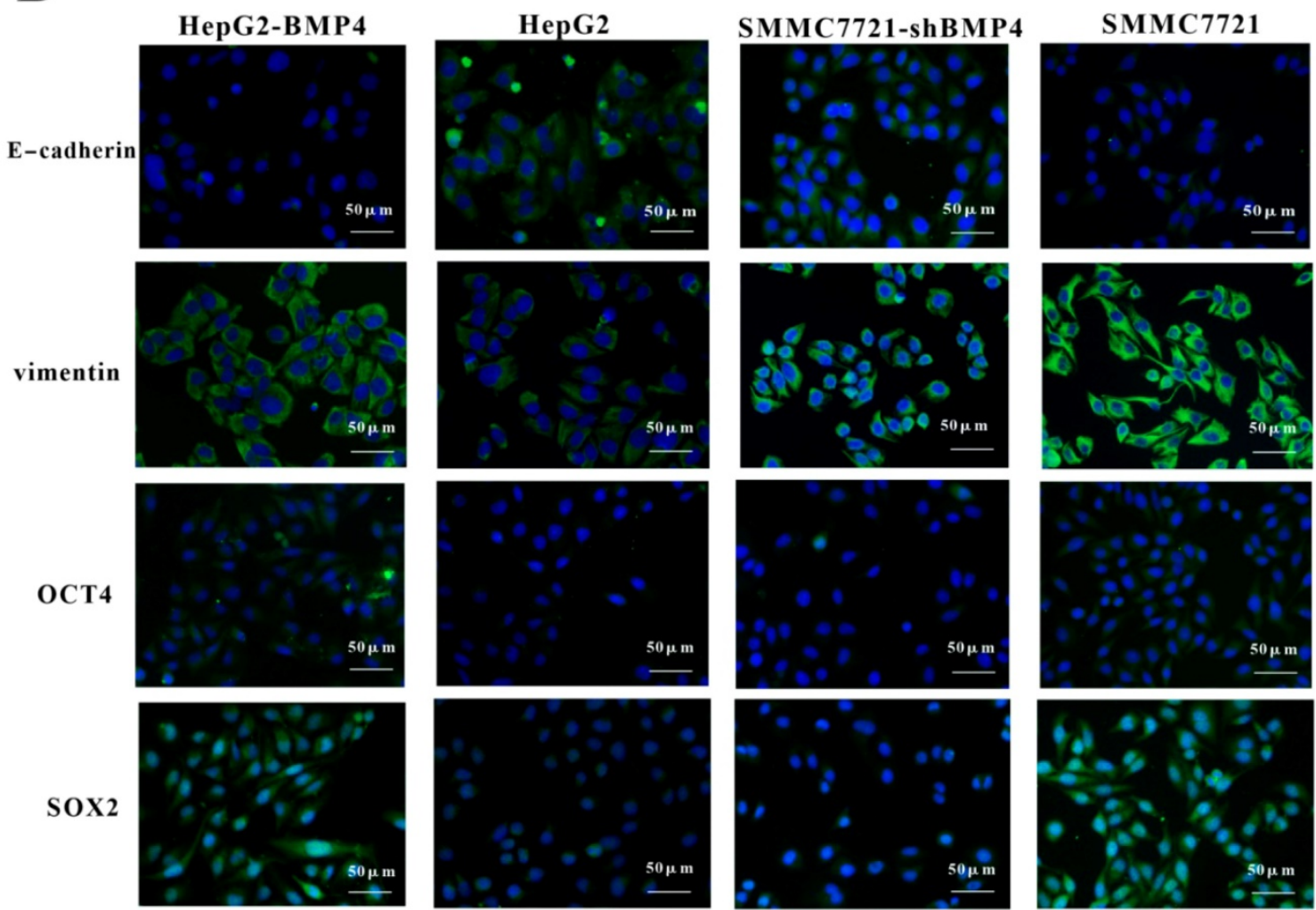

Fig. 5. Expression of E-cadherin, vimentin, OCT4 and SOX2 were evaluated according to western blot (A) and immunofluorescence (B). Cells overexpressing BMP4 had enhanced vimentin, OCT4 and SOX2 expression, but decreased E-cadherin expression compared with the controls; cells under-expressing BMP4 had decreased vimentin, OCT4 and SOX 2 expression, but increased E-cadherin in contrast to the controls. ${ }^{*} \mathrm{P}<0.05$, scale bar $=50 \mu \mathrm{m}$. 

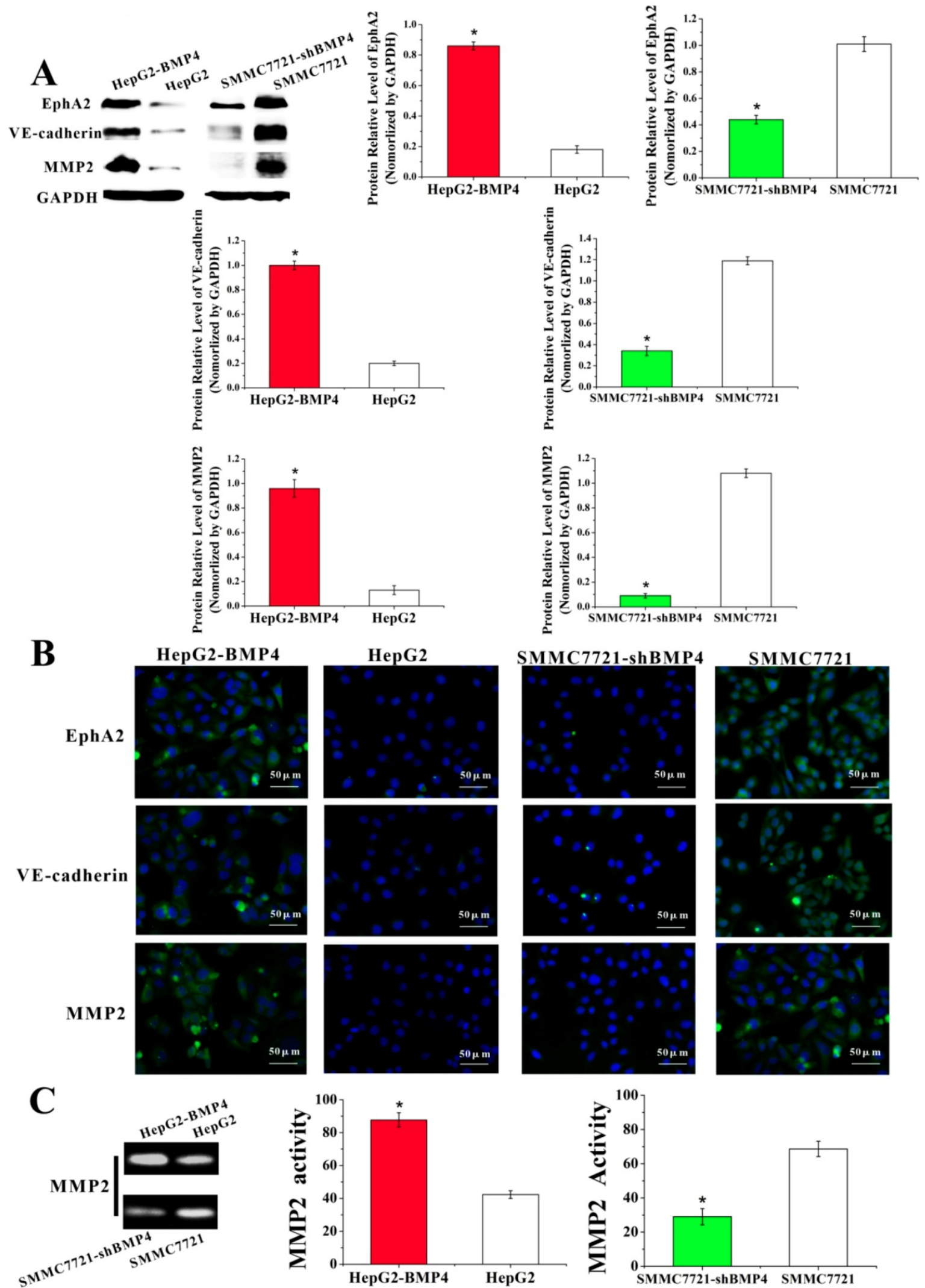

Fig. 6. Expression of EphA2, VE-cadherin and MMP2 were evaluated through western blot (A) and immunofluorescence (B). Cells overexpressing BMP4 had higher EphA2, VE-cadherin and MMP2 protein levels in contrast to controls; cells under-expressing BMP4 had lower EphA2, VE-cadherin and MMP2 protein levels compared with controls, scale bar $=50 \mu \mathrm{m}$. (C) MMP2 activity was analyzed by zymography assay. Stronger MMP2 bands were observed after overexpressing BMP4 in HepG2 cells; weaker MMP2 bands were observed after knocking down BMP4 in SMMC7721 cells. *P<0.05. 


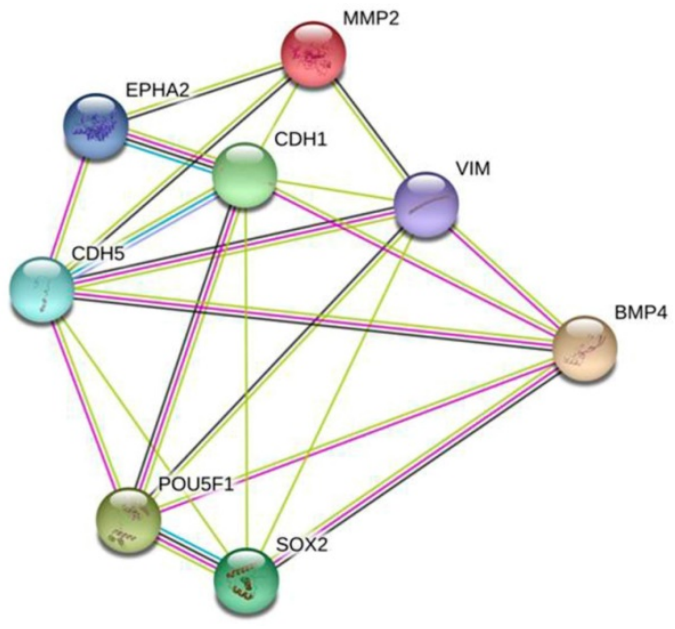

Fig. 7. A predictive relationship between BMP4 and 7 genes related to VM formation. The network showed the interactions between BMP4, VE-cadherin (CDH5), EphA2, MMP2, E-cadherin (CDH1), vimentin (VIM), OCT4 (POU5F1) and SOX2 according to the STRING database. Nodes represent proteins. Colored lines represent the interaction between proteins. Evidence for these interactions is derived from experimental evidence (purple lines), text-mining evidence (green lines), co-expression evidence (black lines), co-occurrence evidence (navy blue lines) and databases evidence (light blue lines).

EphA2, an epithelial receptor and tyrosine kinase, has been shown to be important in the VM formation. VE-cadherin, as a cell adhesion molecule, is also positively associated with VM formation [55]. A lack of VE-cadherin failed to induce VM networks [56]. In the present report, the expression levels of EphA2 and VE-cadherin were increased following BMP4 up-regulation and reduced following BMP4 down-regulation. Our findings were further supported by a positive relationship between BMP4 expression level and MMP2 activity, a downstream protein to EphA2/VE-cadherin signaling that acts as an important part in VM formation [57]. Accumulating evidence from this report implied that EphA2 and VE-cadherin up-regulation activated MMP2 through BMP4, which then promoted cell migration, invasion, and the molding process, thereby causing VM formation. Our results have provided support to the following processes: BMP4 $\rightarrow$ EphA2/VE-cadherin/MMP2 $\rightarrow$ VM. This pathway may be a crucial cascade in the VM formation in HCC.

In conclusion, $\mathrm{VM}$ is a specialized mechanism that supports tumor proliferation and invasiveness but does not respond to available angiogenic inhibitors. Our previous studies and other similar studies have started to uncover the effect of VM formation in cancers and the VM related factors. But the exact mechanism in VM formation is still unclear. On the basis of the effect of VM on hepatocellular carcinoma, we presented in this study a potential mechanism for VM formation in HCC. We have shown that BMP4 was capable of promoting VM formation in HCC through induction of EMT stemness and modulating the EphA2/VE-cadherin/
MMP2 signaling pathway. These findings were further supported by the STRING database. Consequently, BMP4 and its related molecular pathways may be worthy of further investigation and help lay the foundation for better anti-tumor therapies.

\section{Abbreviations}

VM: vasculogenic mimicry; HCC: human hepatocellular carcinoma; EMT: epithelial-mesenchymal transition; MMP2: matrix metalloproteinase 2; BMPs: bone morphogenetic proteins; PBS: phosphate-buffered saline; HRP: horseradish peroxidase; PAS: periodic acid-schiff; FBS: fetal bovine serum; 3D: three-dimensional; SDS-PAGE: sodium dodecyl sulfate polyacrylamide gel electrophoresis; DAPI: 4',6-diamidino-2-phenylindole; SD: standard deviation.

\section{Acknowledgments}

This work was supported by grants from the Key project of the National Natural Science Foundation of China (No. 81230050, No. 81172046 and No. $81173091)$, the Key project of the Tianjin Natural Science Foundation (No. 12JCZDJC23600), and The Cooperation project of China-Sweden (No. 09ZCZDSF04400).

\section{Competing Interests}

The authors have declared that no competing interest exists.

\section{References}

1. Farazi PA, DePinho RA. Hepatocellular carcinoma pathogenesis: from genes to environment. Nat Rev Cancer. 2006;6:674-687.

2. El-Serag HB, Rudolph KL. Hepatocellular carcinoma: epidemiology and molecular carcinogenesis. Gastroenterology. 2007;132:2557-2576.

3. Tung-Ping Poon R, Fan ST, Wong J. Risk factors, prevention, and management of postoperative recurrence after resection of hepatocellular carcinoma. Ann Surg. 2000;232:10-24.

4. Sun B, Zhang S, Zhang D, et al. Vasculogenic mimicry is associated with high tumor grade, invasion and metastasis, and short survival in patients with hepatocellular carcinoma. Oncol Rep. 2006;16:693-698.

5. Maniotis AJ, Folberg R, Hess A, et al. Vascular channel formation by human melanoma cells in vivo and in vitro: vasculogenic mimicry. Am J Pathol. 1999;155:739-752.

6. Folberg R, Hendrix MJ, Maniotis AJ. Vasculogenic mimicry and tumor angiogenesis. Am J Pathol. 2000;156:361-381.

7. Gou JQ, Zheng $\mathrm{OH}$, Chen $\mathrm{H}$, et al. Ginsenoside $\mathrm{Rg} 3$ inhibition of vasculogenic mimicry in pancreatic cancer through downregulation of VE-cadherin/EphA2/MMP9/MMP2 expression. Int J Oncol. 2014;45:1065-1072.

8. Ding YP, Yang $\mathrm{XD}, \mathrm{Wu} \mathrm{Y}$, et al. Autophagy promotes the survival and development of tumors by participating in the formation of vasculogenic mimicry. Onclo Rep. 2014;31:2321-2327.

9. Wang $\mathrm{H}$, Lin $\mathrm{H}$, Pan J, et al. Vasculogenic mimicry in Prostate Cancer: The Roles of EphA2 and PI3K. J Cancer. 2016;7:1114-1124.

10. Sun T, Zhao N, Zhao XL, et al. Expression and functional significance of Twist1 in hepatocellular carcinoma: its role in vasculogenic mimicry. Hepatology. 2010;51:545-556.

11. Sun B, Zhang D, Zhao N, et al. Epithelial-to-endothelial transition and cancer stem cells: two cornerstones of vasculogenic mimicry in malignant tumors. Oncotarget. 2017:8:30502-30510.

12. Hendrix MJ, Seftor EA, Hess AR, et al. Vasculogenic mimicry and tumour-cell plasticity: lessons from melanoma. Nat Rev Cancer. 2003;3:411-421. 
13. Hess AR, Seftor EA, Seftor RE, et al. Phosphoinositide 3-kinase regulates membrane Type 1-matrix metalloproteinase (MMP) and MMP-2 activity during melanoma cell vasculogenic mimicry. Cancer Res. 2003;63:4757-4762.

14. Hogan BL. Bone morphogenetic proteins in development. Curr Opin Genet Dev. 1996;6:432-438.

15. Miyazawa K, Shinozaki M, Hara T, et al. Two major Smad pathways in TGF-beta superfamily signalling. Genes Cells. 2002;7:1191-1204.

16. Hatakeyama S, Gao YH, Ohara-Nemoto Y, et al. Expression of bone morphogenetic proteins of human neoplastic epithelial cells. Biochem Mol Biol Int. 1997;42:497-505

17. Hatakeyama S, Ohara-Nemoto Y, Kyakumoto S, et al. Expression of bone morphogenetic protein in human adenocarcinoma cell line. Biochem Biophys Res Commun. 1993;190:695-701.

18. Ide $\mathrm{H}$, Yoshida $\mathrm{T}$, Matsumoto N, et al. Growth regulation of human prostate cancer cells by bone morphogenetic protein-2. Cancer Res. 1997;57:5022-5027.

19. Kleeff J, Maruyama H, Ishiwata T, et al. Bone morphogenetic protein 2 exerts diverse effects on cell growth in vitro and is expressed in human pancreatic cancer in vivo. Gastroenterology. 1999;116:1202-1216.

20. Kiyozuka $Y$, Nakagawa H, Senzaki H, et al. Bone morphogenetic protein-2 and type IV collagen expression in psammoma body forming ovarian cancer. Anticancer Res. 2001;21:1723-1730.

21. Xia Y, Babitt JL, Sidis Y, et al. Hemojuvelin regulates hepcidin expression via a selective subset of BMP ligands and receptors independently of neogenin. Blood. 2008;111:5195-5204.

22. Rojas A, De Val S, Heidt AB, et al. Gata4 expression in lateral mesoderm is downstream of BMP4 and is activated directly by Forkhead and GATA transcription factors through a distal enhancer element. Development. 2005;132:3405-3417.

23. Shin D, Shin $\mathrm{CH}$, Tucker J, et al. Bmp and Fgf signaling are essential for liver specification in zebrafish. Development. 2007;134:2041-2050.

24. Rossi JM, Dunn NR, Hogan BL, et al. Distinct mesodermal signals, including BMPs from the septum transversum mesenchyme, are required in combination for hepatogenesis from the endoderm. Genes Dev. 2001;15:1998-2009.

25. Rothhammer T, Bataille F, Spruss T, et al. Functional implication of BMP4 expression on angiogenesis in malignant melanoma. Oncogene. 2007;26:4158-4170.

26. Maegdefrau U, Amann T, Winklmeier A, et al. Bone morphogenetic protein 4 is induced in hepatocellular carcinoma by hypoxia and promotes tumour progression. J Pathol. 2009;218:520-529.

27. Ketolainen JM, Alarmo EL, Tuominen VI, et al. Parallel inhibition of cell growth and induction of cell migration and invasion in breast cancer cells by bone morphogenetic protein 4. Breast Cancer Res Treat. 2010;124:377-386.

28. Deng H, Makizumi R, Ravikumar TS, et al. Bone morphogenetic protein- 4 is overexpressed in colonic adenocarcinomas and promotes migration and invasion of HCT116 cells. Exp Cell Res. 2007;313:1033-1044

29. Zhou K, Shi X, Huo J, et al. Bone morphogenetic protein 4 is overexpressed in and promotes migration and invasion of drug-resistant cancer cells. Int J Biol Macromol. 2017;101:427-437.

30. Guo X, Xiong L, Zou L, et al. Upregulation of bone morphogenetic protein 4 is associated with poor prognosis in patients with hepatocellular carcinoma. Pathol Oncol Res. 2012:18:635-640.

31. Hamada S, Satoh K, Hirota M, et al. Bone morphogenetic protein 4 induces epithelial-mesenchymal transition through MSX2 induction on pancreatic cancer cell line. J Cell Physiol. 2007;213:768-774.

32. Kotiyal S, Bhattacharya S. Epithelial Mesenchymal Transition and Vascular Mimicry in Breast Cancer Stem Cells. Crit Rev Eukaryot Gene Expr. 2015;25:269-280.

33. Ma J, Zeng S, Zhang Y, et al. BMP4 promotes oxaliplatin resistance by an induction of epithelial-mesenchymal transition via MEK1/ERK/ELK1 signaling in hepatocellular carcinoma. Cancer Lett. 2017;411:117-129.

34. Martínez VG, Rubio C, Martínez-Fernández M, et al. BMP4 Induces M2 Macrophage Polarization and Favors Tumor Progression in Bladder Cancer. Clin Cancer Res. 2017;23:7388-7399.

35. Blick T, Hugo H, Widodo $\mathrm{E}$, et al. Epithelial mesenchymal transition traits in human breast cancer cell lines parallel the CD44(hi/)CD24(lo/-) stem cell phenotype in human breast cancer. J Mammary Gland Biol Neoplasia. 2010;15:235-252.

36. Zeng S, Zhang Y, Ma J, et al. BMP4 promotes metastasis of hepatocellular carcinoma by an induction of epithelial-mesenchymal transition via upregulating ID2. Cancer Lett. 2017;390:67-76.

37. Rahman MA Dhar DK, Yamaguchi $\mathrm{E}$, et al Coexpression of inducible nitric oxide synthase and COX-2 in hepatocellular carcinoma and surrounding liver: possible involvement of COX-2 in the angiogenesis of hepatitis C virus-positive cases. Clin Cancer Res. 2001;7:1325-1332.

38. Sun B, Qie S, Zhang $\mathrm{S}$, et al. Role and mechanism of vasculogenic mimicry in gastrointestinal stromal tumors. Hum Pathol. 2008; 39:444-451.

39. Sun $\mathrm{T}$, Sun $\mathrm{BC}$, Zhao $\mathrm{XL}$, et al. Promotion of tumor cell metastasis and vasculogenic mimicry by way of transcription coactivation by $\mathrm{Bcl}-2$ and Twist1: a study of hepatocellular carcinoma. Hepatology. 2011;54:1690-1706

40. Iwatsuki M, Mimori K, Yokobori T, et al. Epithelial-mesenchymal transition in cancer development and its clinical significance. Cancer Sci. 2010-101-293-299.

41. Santisteban M, Reiman JM, Asiedu MK, et al. Immune-induced epithelial to mesenchymal transition in vivo generates breast cancer stem cells. Cancer Res. 2009;69:2887-2895
42. Mani SA, Guo W, Liao MJ, et al. The epithelial-mesenchymal transition generates cells with properties of stem cells. Cell. 2008;133:704-715.

43. Ganju P, Shigemoto K, Brennan J, et al. The Eck receptor tyrosine kinase is implicated in pattern formation during gastrulation, hindbrain segmentation and limb development. Oncogene. 1994;9:1613-1624.

44. Hess AR, Seftor EA, Gardner LM, et al. Molecular regulation of tumor cell vasculogenic mimicry by tyrosine phosphorylation: role of epithelial cell kinase (Eck/EphA2). Cancer Res. 2001;61:3250-3255.

45. Margaryan NV, Strizzi L, Abbott DE, et al. EphA2 as a promoter of melanoma tumorigenicity. Cancer Biol Ther. 2009;8:279-288.

46. Gory-Faure S, Prandini MH, Pointu $\mathrm{H}$, et al. Role of vascular endothelial-cadherin in vascular morphogenesis. Development. 1999;126:2093-2102.

47. Seftor RE, Seftor EA, Koshikawa N, et al. Cooperative interactions of laminin 5 gamma2 chain, matrix metalloproteinase-2, and membrane type-1-matrix/metalloproteinase are required for mimicry of embryonic vasculogenesis by aggressive melanoma. Cancer Res. 2001;61:6322-6327.

48. Folberg R, Maniotis AJ. Vasculogenic mimicry. APMIS. 2004:112:508-525

49. Folberg R, Rummelt V, Parys-Van Ginderdeuren R, et al. The prognostic value of tumor blood vessel morphology in primary uveal melanoma. Ophthalmology. 1993;100:1389-1398.

50. Makitie T, Summanen P, Tarkkanen A, et al. Microvascular loops and networks as prognostic indicators in choroidal and ciliary body melanomas. J Natl Cancer Inst. 1999;91:359-367.

51. Thiery JP. Epithelial-mesenchymal transitions in development and pathologies. Curr Opin Cell Biol. 2003;15:740-746.

52. Thiery JP. Epithelial-mesenchymal transitions in tumour progression. Nat Rev Cancer. 2002;2:442-454.

53. Huber MA, Kraut N, Beug H. Molecular requirements for epithelial-mesenchymal transition during tumor progression. Curr Opin Cell Biol. 2005;17:548-558.

54. Marotta LL, Polyak K. Cancer stem cells: a model in the making. Curr Opin Genet Dev. 2009;19:44-50.

55. Zhang S, Zhang D, Sun B. Vasculogenic mimicry: current status and future prospects. Cancer Lett. 2007;254:157-164.

56. Hendrix MJ, Seftor EA, Meltzer PS, et al. Expression and functional significance of VE-cadherin in aggressive human melanoma cells: role in vasculogenic mimicry. Proc Natl Acad Sci U S A. 2001;98:8018-8023.

57. Pandey A, Lazar DF, Saltiel AR, et al. Activation of the Eck receptor protein tyrosine kinase stimulates phosphatidylinositol 3-kinase activity. J Biol Chem. 1994;269:30154-30157. 